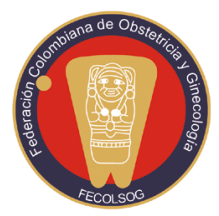

Revista Colombiana de Obstetricia y Ginecología Vol. 70 No. $3 \bullet J u l i o-S e p t i e m b r e ~ 2019 \bullet(181-188)$

\title{
SEGURIDAD DEL MANEJO LAPAROSCÓPICO DE PACIENTES CON ENDOMETRIOSIS INFILTRATIVA PROFUNDA, EN UN CENTRO DE REFERENCIA EN PEREIRA, COLOMBIA. COHORTE RETROSPECTIVA, 2007-2016
}

Safety of the laparoscopic management of patients with deep infiltrative endometriosis in a reference center in Pereira, Colombia. Retrospective cohorte, 2007-2016

Claudia Patricia Zambrano-Moncayo, MD ${ }^{* 1}$; José Duván López-Jaramillo, MD $^{1}$; Jorge Darío López-Isanoa, $\mathrm{MD}^{1}$; Ana Lucía Herrera-Betancourt, $\mathrm{MD}^{2}$; Lina María Zuluaga-García, $M D^{1}$; Danny Leandro Piedrahíta-Gutiérrez, MD $^{I}$; Juan José Montoya-Martínez, MD, MSc ${ }^{3}$; Claudia Bastidas-Guarín, $M^{4}$; Juan Diego Villegas-Echeverri, $M D^{5}$

Recibido: diciembre 09/18 - Aceptado: octubre 09/19

\section{RESUMEN}

Objetivo: describir los hallazgos intraoperatorios, los procedimientos realizados y la seguridad del manejo quirúrgico por vía laparoscópica de la endometriosis infiltrativa profunda (EIP).

Materiales y métodos: cohorte histórica descriptiva. Ingresaron pacientes con sospecha diagnóstica

* Correspondencia: Claudia Patricia Zambrano Moncayo, Cra. 19a \# 107-41 apto. 204, Bogotá (Colombia). claupzm@gmail.com

1 Especialista en Ginecología y Obstetricia, cirujano laparoscopista, Unidad ALGIA-Clínica Comfamiliar, Pereira (Colombia).

2 Especialista en Ginecología y Obstetricia, cirujana laparoscopista, Unidad ALGIA-Clínica Comfamiliar, Pereira (Colombia). Unidad de Dolor Pélvico Crónico Sanitas, Bogotá (Colombia).

3 Médico y cirujano, maestría en Epidemiología, Unidad de Investigación y Docencia, Salud Comfamiliar, Pereira (Colombia).

4 Especialista en Ginecología y Obstetricia, Fellowship Cirugía Laparoscópica y Dolor Pélvico Crónico; epidemióloga. AAGL-Clínica Comfamiliar, Pereira (Colombia).

5 Especialista en Ginecología y Obstetricia, cirujano laparoscopista, director Científico Unidad ALGIA-Clínica Comfamiliar, Pereira (Colombia). prequirúrgica de EIP por hallazgos clínicos, ultrasonido pélvico o imágenes de resonancia magnética y con confirmación histológica de la enfermedad. Las pacientes fueron llevadas a cirugía mínimamente invasiva entre 2007 y 2016, en una institución de salud de referencia ubicada en Pereira, Colombia. Se evaluaron variables sociodemográficas, clínicas, hallazgos intraoperatorios, tipos de procedimientos realizados, complicaciones intra y posoperatorias y control del dolor a las 6 semanas. Se hace un análisis descriptivo.

Resultados: se incluyeron 167 pacientes. La localización más frecuente de la enfermedad fue el tabique recto-vaginal (85,7\%). Un total de 83 pacientes $(49,7 \%)$ presentaron endometriosis intestinal. De estas, al 86\% se les realizó shaving (afeitado o nodulectomía), y al 13,2\% resección intestinal segmentaria. Un total de 4 pacientes (2,4\%) presentaron complicaciones intraoperatorias, de las cuales una 
requirió laparoconversión y otras 5 pacientes (2,9\%) desarrollaron complicaciones posoperatorias.

Conclusiones: el manejo laparoscópico de la EIP es una alternativa por considerar, con una frecuencia de complicaciones cercana al $5 \%$. Se requieren estudios aleatorizados con grupo control para una mejor evaluación de la seguridad y eficacia.

Palabras clave: endometriosis; laparoscopia; cuidados posoperatorios; dolor pélvico; calidad de vida.

\section{ABSTRACT}

Objective: Describe the intraoperative findings, procedures and the safety of laparoscopic surgical management of Deep Infiltration Endometriosis (DIE).

Materials and methods: A descriptive historical cohort study of patients with suspected pre-surgical diagnosis of DIE due to clinical findings, pelvic ultrasound or magnetic resonance imaging and histological confirmation of the disease. The patients were taken to minimally invasive surgery between 2007 and 2016 in a reference health institution located in Pereira, Colombia. Sociodemographic, clinical, intraoperative findings, types of procedure performed, intra and postoperative complications and post-surgical pain levels at 6 weeks were evaluated. A descriptive analysis was performed.

Results: One hundred and sixty seven patients were included. The most frequent location of the disease was the recto-vaginal septum (85.7\%). A total of 83 patients (49.7\%) had bowel endometriosis. Of these, $86 \%$ had a shaving and $13.2 \%$ segmental bowel resection. Four patients (2.4\%) had intraoperative complications. One was converted to laparotomy and other five (2.9\%) had post-surgical infection.

Conclusions: Laparoscopic management of DIE is an option to be considered with a 5\% of complication frequency. Randomized studies with a control group are required for a better evaluation of safety and efficacy.
Key words: Endometriosis; laparoscopy; postoperative care; pelvic pain; quality of life.

\section{INTRODUCCIÓN}

La endometriosis infiltrativa profunda (EIP) se define como la presencia de adenomiosis externa con glándulas endometriales y estroma en tejido fibromuscular que infiltra órganos y estructuras anatómicas, independientemente de la profundidad $(1,2)$. Es la forma más severa de la endometriosis y se encuentra en el 4 al $37 \%$ de las pacientes con endometriosis $(3,4)$ con una edad promedio de presentación de 31 años (5). Las estructuras más frecuentemente comprometidas son los ligamentos uterosacros (40-45\%), el torus uterino (23-35\%), el tabique recto-vaginal (18-89\%), el intestino (7$27 \%)$ y la vía urinaria $(6 \%)(6,7,8)$. La EIP se asocia con dolor pélvico crónico e infertilidad y tiene un impacto importante en la calidad de vida de las mujeres (9). En Estados Unidos, los costos directos asociados a la endometriosis se han estimado en US\$ 12.118 por paciente/año, y los indirectos en US\$ 15.737 por paciente/año $(10,11)$.

El diagnóstico inicial y la planeación del manejo se basan en los hallazgos clínicos e imagenológicos, pero el definitivo lo confirma el estudio histopatológico $(2,12,13)$. Los síntomas asociados son dolor pélvico incapacitante (28-37\%), dismenorrea (86$94 \%)$, dispareunia profunda (69-59\%), disquecia (30-35\%), diarrea menstrual o estreñimiento, rectorragia o infertilidad (23-50\%) $(1,14)$. El examen físico tiene sensibilidad (S) de $72 \%$ y especificidad (E) de 54\% para el diagnóstico de lesiones en rectosigmoides y una S de $68 \%$ y E del $46 \%$ para las lesiones retrocervicales (2). La ecografía transvaginal con preparación intestinal y medio de contraste tiene una $\mathrm{S}$ entre $59-85 \%$ y una $\mathrm{E}$ entre $86-97 \%$ para detección de lesiones en recto sigmoides, tabique recto-vaginal y ligamentos uterosacros $(12,15)$. A su vez, las imágenes de resonancia magnética (RM) tienen una $\mathrm{S}$ entre $66-85 \%$ y una $\mathrm{E}$ entre $93-97 \%$ para detección de lesiones de $\operatorname{EIP}(2,9)$. 
El tratamiento de la EIP puede ser médico o quirúrgico. El manejo médico hormonal es la primera consideración, es eficiente y bien tolerado para disminuir los síntomas dolorosos en el 70 al $100 \%(16,17)$. La cirugía está indicada en pacientes que no responden a manejo médico previo y debe basarse en factores como la edad de la paciente, la intensidad del dolor (escala visual análoga mayor a 7), riesgo de obstrucción intestinal y deseo de fertilidad (9) así como mejoría en la calidad de vida y la función sexual (17-21).

Algunos autores informan que la laparoscopia tiene más beneficios ya que provee un mejor acceso a los espacios retroperitoneales y una mejor visualización del campo operatorio, lo que permite la apropiada delimitación del tejido comprometido para lograr una adecuada resección (22); reducción en el uso de analgésicos en el posoperatorio inmediato, menor sangrado, menor tiempo intrahospitalario, menor frecuencia en las complicaciones relacionadas con la herida quirúrgica y menor incapacidad $(5,13)$.

Es poca la literatura regional sobre el uso de la laparoscopia en el manejo de mujeres con EIP, los reportes provienen principalmente de Brasil (23) y de Colombia (22). Dado el frecuente compromiso profundo de las estructuras adyacentes es importante aportar datos sobre la seguridad del abordaje laparoscópico en el tratamiento quirúrgico de la entidad. Por tanto, el objetivo del presente estudio es describir los hallazgos intraoperatorios, los procedimientos realizados, las complicaciones intra y posoperatorias tempranas del manejo por vía laparoscópica de la EIP en un grupo de pacientes llevadas a cirugía, como también hacer una aproximación a su eficacia en el manejo de la entidad.

\section{MATERIALES Y MÉTODOS}

Diseño y población. Cohorte histórica descriptiva de mujeres con diagnóstico EIP llevadas a manejo quirúrgico por vía laparoscópica. El estudio se desarrolló en la Unidad de Laparoscopia Ginecoló- gica Avanzada y Dolor Pélvico (ALGIA), centro de referencia de dolor pélvico crónico y laparoscopia avanzada, ubicado en la Clínica Comfamiliar en Pereira, Colombia, entre 2007 y 2016, que atiende población afiliada al régimen contributivo y subsidiado por el Estado y pacientes privadas en el Sistema de seguridad social en Colombia. Se hizo muestreo consecutivo y se intentó la inclusión de todas las intervenidas en el periodo de estudio.

Procedimiento. Las pacientes fueron intervenidas por el grupo multidisciplinario de cirugía mínimamente invasiva y cirujano general entrenados en resección de endometriosis profunda. Fueron identificadas por el código de diagnóstico CIE-10 desde el N800 hasta el N809 a partir de una base de datos de la unidad. Posteriormente, se confirmó el diagnostico de EIP ya fuera por el reporte de lesiones infiltrativas en región pélvica en la RM, o por ultrasonido transvaginal de mapeo de IP con preparación intestinal y medio de contraste vaginal; adicionalmente, a todas se les confirmo el resultado de patología positiva para EIP en el control posoperatorio a los 10 días. Para medir la intensidad de los síntomas, se utilizó la escala visual análoga (EVA), donde 0 representa la ausencia de síntomas y 10 el grado más severo de intensidad de los síntomas dolorosos, esta fue registrada por cada paciente antes de la cirugía y a las 6 semanas. Se creó una base de datos en Excel con un formulario de recolección de datos previamente diseñado, validado por tres expertos clínicos y probado mediante una prueba piloto ejecutada sobre 15 historias clínicas, donde se registraron las variables de cada una de las pacientes. Se realizó verificación de la calidad por medio del estadístico Stata versión 14.0

Las variables medidas fueron: edad, estado civil, tipo de afiliación al sistema de seguridad social, antecedentes ginecobstétricos y antecedentes quirúrgicos, síntomas asociados antes de la cirugía (dismenorrea, dispareunia, dolor pélvico crónico, disquecia y síntomas urinarios). También se determinaron las características del procedimiento, los 
hallazgos quirúrgicos, la localización de las lesiones, los procedimientos realizados, la estancia hospitalaria, la presencia de complicaciones intraoperatorias según la clasificación Clavien Dindo (Escala de morbildad basada en las consecuencias terapéuticas de las complicaciones) (24) y la severidad del dolor según la escala análoga visual antes y seis semanas después de la cirugía en las pacientes a quienes se les aplicó dicha escala.

Análisis estadístico. Se utilizó estadística descriptiva. Para las variables cuantitativas se calculó la media y la desviación estándar (DE) y para las cualitativas se utilizaron tablas de frecuencias. Para calcular diferencias entre el dolor inicial y el dolor a las 6 semanas se aplicó estadística no paramétrica Wilconson. Como valor de significancia estadística se tomó un valor de p menor a 0,05. Para el procesamiento de datos se utilizó SPSS versión 14.0

Aspectos éticos. Este estudio fue aprobado por el comité de ética de la Clínica Comfamiliar Risaralda de Pereira (Acta No. 000013). Se garantizó la confidencialidad y privacidad de la información.

\section{RESULTADOS}

Según los datos suministrados por la oficina de Estadística de la Clínica Comfamiliar, entre 2007 y 2016 se presentaron 24.783 atenciones con diagnóstico de patología benigna ginecológica. De estas, 1.473 (5,9\%) tenían diagnóstico de endometriosis (Código CIE-10 N800 - N809), de las cuales 169 $(11,4 \%)$ fueron llevadas a cirugía laparoscópica; en 2 no se dispuso del resultado de patología quirúrgica por lo que no fueron incluidas y, finalmente, quedaron 167 pacientes en el estudio; 27 de ellas no asistieron a control, por lo que se tuvo disponibilidad de los datos de 140 pacientes $(83,8 \%)$ durante el seguimiento a las 6 semanas.

El diagnostico se realizó con ecografía transvaginal con preparación intestinal y medio de contraste vaginal en el $65 \%$ de las pacientes, RM en el $15 \%$ de las pacientes y al $11 \%$ se les realizaron ambas imágenes diagnósticas. El 9\% no tenía imágenes y el diagnóstico final fue solo por los hallazgos operatorios y de histopatología. La media de edad fue de 31,5 años (DE \pm 7 ). Un total de 55 (32,9\%) pacientes tenían pareja estable, 160 (95,8\%) residían en área urbana y 117 (70\%) pertenecían al régimen contributivo. Los síntomas que se presentaron con mayor frecuencia fueron la dismenorrea en 146 (87,4\%) y la dispareunia profunda en 122 (73\%). Respecto al dolor, la EVA inicial fue igual o mayor a 7 en 111 (80,4\%) de 140 pacientes, con una media de 7,3 (DE $\pm 2,6)$. En 65 pacientes se palparon nódulos en el examen físico inicial, la localización más frecuente fueron los ligamentos uterosacros (LUS) en 102 (61\%) y el torus uterino en $26(15,56 \%)$.

En cuanto a los hallazgos intraoperatorios, las lesiones encontradas con mayor frecuencia durante la cirugía fueron los nódulos del tabique rectovaginal en 143 pacientes $(85,6 \%)$, ligamentos uterosacros en 94 (56,2\%), endometriosis intestinal en 83 (49,7\%), endometriomas bilaterales en 58 (34,7\%) y en una menor proporción la endometriosis del tracto urinario en 16 pacientes (9,5\%), de las cuales en 8 se evidenció endometriosis vesical. El tamaño promedio de los nódulos del tabique recto-vaginal y uterosacros fue de 36,56 mm (DE $\pm 11,3$ ).

Los procedimientos que se realizaron con mayor frecuencia fueron la ureterolisis $(65,2 \%)$, y la cistoscopia (58,6\%). De las 83 pacientes con endometriosis intestinal a 72 se les realizó shaving (afeitado o nodulectomía) intestinal y a 11 resección intestinal segmentaria; de estas 11 pacientes, 7 tenían lesiones en el sigmoides y 4 en el recto. A 5 de las 11 pacientes llevadas a resección intestinal se les realizó ileostomía de protección; 6 pacientes tenían lesiones multifocales. El tamaño promedio de las lesiones intestinales fue de $34 \mathrm{~mm}(\mathrm{DE} \pm 16,7)$.

Un total de 4 pacientes 2,4\% presentaron complicaciones intraoperatorias. De ellas, una paciente tuvo lesión en el recto con diagnóstico intraoperatorio, se realizó reparación vía laparoscópica, sin presentar complicaciones adicionales. Una paciente 
presentó filtración del shaving (afeitado o nodulectomía), que requirió lavado por laparoscopia. Otra paciente presentó epiplocele sintomático que requirió manejo quirúrgico. Se realizó laparoconversión en una paciente $(0,59 \%)$ que tenía antecedente de cinco laparoscopias, y se encontraron múltiples adherencias densas de recto a pared posterior del útero sin lograr una adecuada visualización de los uréteres.

Un total de 5 (2,9\%) pacientes presentó complicaciones posoperatorias: cuatro pacientes $(2,4 \%)$ presentaron abscesos pélvicos en los primeros 7 días posoperatorios, de las cuales tres fueron llevadas a laparoscopia y lavados, y una paciente a lavado por laparotomía. Una más presentó tromboembolismo pulmonar, que requirió manejo intrahospitalario y recibió manejo médico con anticoagulación con enoxaparina por 6 semanas y terapia respiratoria, con posterior evolución clínica favorable. Dos pacientes presentaron fiebre de causa no clara (tabla 1). Las complicaciones se clasificaron, según Clavien Dindo, como III b. No hubo mortalidad durante el procedimiento ni en el seguimiento posoperatorio (tabla 1).

Con respecto a la valoración de la escala de EVA, en las 140 pacientes evaluadas antes de la cirugía se encontró una media de dolor de 7,3 (DS $\pm 2,6$ ), y a las 6 semanas posquirúrgicas se encontró una media de dolor de 1,9 (DS $\pm 1,7)$ diferencia significativa $(\mathrm{p}=0,000)$.

\section{DISCUSIÓN}

Este estudio describe los desenlaces clínicos del manejo quirúrgico laparoscópico de 167 pacientes con EIP, en un periodo de 9 años. Estas corresponden al $11,4 \%$ de las pacientes que consultaron a la unidad por endometriosis en el periodo de observación del estudio. La frecuencia de complicaciones fue del 5,3\% en las pacientes que asistieron a control; se tuvo una frecuencia de laparoconversión de 0,59\%.

Nuestros hallazgos respecto a la frecuencia de la EIP se encuentran dentro del rango reportado en la literatura $(3,4)$. Acerca de la localización en nuestro estudio, estas se ubicaron mayormente en el tabique recto-vaginal, seguido por los ligamentos úterosacros y el intestino, similares a lo informado por Kwok (25) y por Castañeda (22), y diferentes a lo publicado por Chapron (5), quien reporta que la localización más frecuente de las lesiones es en los ligamentos uterosacros (83\%), seguido por el tabique recto-vaginal (56\%) e intestino (55\%); La diferencia se podría explicar porque en nuestra cohorte el diagnóstico fue hecho por ecografía e IRM que tienen una adecuada especificidad para las lesiones en el tabique recto-vaginal (2).

En cuanto a las complicaciones, nuestros hallazgos son similares a las presentadas por Abrao (9), que reportan complicaciones posteriores al manejo quirúrgico laparoscópico del $4 \%$, que

\begin{tabular}{|l|l|l|} 
Tabla 1. & \\
\multicolumn{2}{|c|}{$\begin{array}{l}\text { Complicaciones intra y posquirúrgicas en mujeres con endometriosis profunda sometidas } \\
\text { a laparoscopia en una clínica de referencia en Pereira, Colombia, 2007-2016 }\end{array}$} \\
\hline Complicaciones & N=11 Clavien Dindo \\
\hline Lesión de recto & 1 & III b \\
\hline Filtración del afeitado & 1 & III b \\
\hline Epiplocele & 1 & III b \\
\hline Laparoconversión & 1 & \\
\hline Fiebre & 2 & I \\
\hline Tromboembolismo pulmonar & 1 & II \\
\hline Hematoma sobreinfectado & 4 & III b \\
\hline
\end{tabular}


pueden llegar hasta el $22 \%$ si se requiere resección intestinal. Este autor informa que las complicaciones pueden ser: fístula (0-14\%), hemorragia (1-11\%), infecciones (1-3\%), laparoconversión (<12\%), disfunción vesical (1-71\%) y disfunción intestinal (1-15\%).

Respecto al control del dolor, nuestros resultados son similares a los informados por Donnez et al. en 500 pacientes llevadas a resección de nódulo de tabique recto-vaginal, donde 242 tuvieron seguimiento por más de 2 años, con disminución importante de los síntomas (26). Chapron reporta una mejoría del dolor de 84,2 \%, de la dismenorrea del $81,2 \%$ y de la dispareunia del $94,1 \%$ posterior al manejo quirúrgico (27).

En cuanto a las limitaciones del estudio, la más importante fue la pérdida de 29 pacientes (17\%) en el seguimiento debido a las características retrospectivas de la cohorte; si se toma en cuenta que la pérdida pudo estar asociada al resultado del tratamiento, se podría haber afectado la estimación de la seguridad y la eficacia. La fortaleza está en el importante número de pacientes intervenidas.

\section{CONCLUSIONES}

El manejo laparoscópico de la EIP surge como una alternativa segura. Se requieren estudios aleatorizados con grupo control para una mejor evaluación de su eficacia en el manejo del dolor, y el efecto en la estancia hospitalaria y los costos a nivel local.

\section{REFERENCIAS}

1. Koninckx PR, Ussia A, Adamyan L, Wattiez A, Donnez J. Deep endometriosis: Definition, diagnosis, and treatment. Fertil Steril. 2012;98(3):564-71. Doi: https://doi.org/10.1016/j.fertnstert.2012.07.1061

2. Bazot M, Daraï E. Diagnosis of deep endometriosis: clinical examination, ultrasonography, magnetic resonance imaging, and other techniques. Fertil Steril. 2017;108(6):886-94. Doi: https://doi.org/10.1016/j. fertnstert.2017.10.026

3. Exacoustos C, Malzoni M, Di Giovanni A, Lazzeri L, Tosti C, Petraglia F, et al. Ultrasound mapping system for the surgical management of deep infiltrating endometriosis. Fertil Steril. 2014;102(1). Doi: https:// doi.org/10.1016/j.fertnstert.2014.03.043

4. Nassif J, Trompoukis P, Barata S, Furtado A, Gabriel B, Wattiez A. Management of deep endometriosis. Reprod Biomed Online. 2011;23(1):25-33. Doi: https://doi.org/10.1016/j.rbmo.2010.08.012

5. Chapron C, Fauconnier A, Vieira M, Barakat H, Dousset B, Pansini V, et al. Anatomical distribution of deeply infiltrating endometriosis: Surgical implications and proposition for a classification. Hum Reprod. 2003;18(1):157-61. Doi: https://doi.org/10.1093/ humrep/deg009

6. Perelló M, Martínez-Zamora MA, Torres X, Munrós J, Llecha S, De Lazzari E, et al. Markers of deep infiltrating endometriosis in patients with ovarian endometrioma: a predictive model. Eur J Obstet Gynecol Reprod Biol. 2017;209(2015):55-60. Doi: https:// doi.org/10.1016/j.ejogrb.2015.11.024

7. Gordts S, Koninckx P, Brosens I. Pathogenesis of deep endometriosis. Fertil Steril. 2017;108(6):872-85.e1. Doi: https://doi.org/10.1016/j.fertnstert.2017.08.036

8. Audebert A, Petousis S, Margioula-Siarkou C, Ravanos K, Prapas N, Prapas Y. Anatomic distribution of endometriosis: A reappraisal based on series of 1101 patients. Eur J Obstet Gynecol Reprod Biol. 2018;230:36-40. Doi: https://doi.org/10.1016/j. ejogrb.2018.09.001

9. Abrão MS, Petraglia F, Falcone T, Keckstein J, Osuga Y, Chapron C. Deep endometriosis infiltrating the recto-sigmoid: Critical factors to consider before management. Hum Reprod Update. 2015;21(3):329-39. https://doi.org/10.1093/humupd/dmv003

10. Soliman AM, Yang H, Du EX, Kelley C, Winkel C. The direct and indirect costs associated with endometriosis: A systematic literature review. Hum Reprod. 2016;31(4):712-22. Doi: https://doi.org/10.1093/ humrep/dev335

11. Soliman AM, Taylor H, Bonafede M, Nelson JK, Castelli-Haley J. Incremental direct and indirect cost burden attributed to endometriosis surgeries in the United States. Fertil Steril. 2017;107(5):1181-90.e2. Doi: https://doi.org/10.1016/j.fertnstert.2017.03.020 
12. Nisenblat V, Pmm B, Farquhar C, Johnson N, Ml H. Imaging modalities for the non-invasive diagnosis of endometriosis (Review). Cochrane Database Syst Rev. 2016;(2). Doi: https://doi.org/10.1002/14651858. CD009591.pub2

13. Dunselman GAJ, Vermeulen N, Becker C, CalhazJorge C, D'Hooghe T, De Bie B, et al. ESHRE guideline: Management of women with endometriosis. Hum Reprod. 2014;29(3):400-12. Doi: https://doi. org/10.1093/humrep/det457

14. Barcellos MB, Lasmar B, Lasmar R. Agreement between the preoperative findings and the operative diagnosis in patients with deep endometriosis. Arch Gynecol Obstet. 2016;293(4):845-50. Doi: https:// doi.org/10.1007/s00404-015-3892-x

15. Guerriero S, Saba L, Pascual MA, Ajossa S, Rodriguez I, Mais V, et al. Transvaginal ultrasound (TVS) versus Magnetic Resonance (MR) for diagnosing deep infiltrating endometriosis: a systematic review and meta-analysis. Ultrasound Obstet Gynecol. 2018;51(January 2016):586-95. Doi: https://doi. org/10.1002/uog.18961

16. Practice Committee of the American Society for Reproductive Medicine. Treatment of pelvic pain associated with endometriosis. Fertil Steril. 2006;86(5 Suppl 1):S18-27. Doi: https://doi.org/10.1016/j. fertnstert.2006.08.072

17. Ferrero S, Alessandri F, Racca A, Leone Roberti Maggiore U. Treatment of pain associated with deep endometriosis: Alternatives and evidence. Fertil Steril. 2015;104(4):771-92. Doi: https://doi.org/10.1016/j. fertnstert.2015.08.031

18. Chopin N, Vieira M, Borghese B, Foulot H, Dousset B, Coste J, et al. Operative management of deeply infiltrating endometriosis: Results on pelvic pain symptoms according to a surgical classification. J Minim Invasive Gynecol. 2005;12(2):106-12. Doi: https://doi.org/10.1016/j.jmig.2005.01.015

19. Ribeiro PAA, Sekula VG, Abdalla-Ribeiro HS, Rodrigues FC, Aoki T AJ. Impact of laparoscopic colorectal segment resection on quality of life in women with deep endometriosis: one year follow-up. Qual Life Res Int J
Qual Life Asp Treat Care Rehabil. 2014;23(2):639-43. Doi: https://doi.org/10.1007/s11136-013-0481-y

20. Bassi MA, Podgaec S, Dias JA, D’Amico Filho N, Petta CA, Abrao MS. Quality of life after segmental resection of the rectosigmoid by laparoscopy in patients with deep infiltrating endometriosis with bowel involvement. J Minim Invasive Gynecol. 2011;18(6):730-3. DOI: https://doi.org/10.1016/j.jmig.2011.07.014

21. Ford J, English J, Miles WA, Giannopoulos T. Pain, quality of life and complications following the radical resection of rectovaginal endometriosis. BJOG An Int J Obstet Gynaecol. 2004;111(4):353-6. Doi: https://doi. org/10.1111/j.1471-0528.2004.00093.x

22. Castañeda J, Almanza L, Muñoz J, Arango A, De los Rios JF, Serna E, et al. Endometriosis profunda infiltrante: seguimiento a un año luego de tratamiento laparoscopico. Rev Colomb Obstet Ginecol. 2010;61(2):129-35.

23. Ribeiro PAA, Sekula VG, Abdalla-Ribeiro HS, Rodrigues FC, Aoki T, Aldrighi JM. Impact of laparoscopic colorectal segment resection on quality of life in women with deep endometriosis: one year follow-up. Qual Life Res. 2014;23(2):639-43. DOI: https://doi.org/10.1007/ s11136-013-0481-y

24. Dindo D, Demartines N, Clavien PA. Classification of surgical complications: A new proposal with evaluation in a cohort of 6336 patients and results of a survey. Ann Surg. 2004;240(2):205-13. Doi: https://doi. org/10.1097/01.sla.0000133083.54934.ae

25. Kwok A, Lam FA, Ford R. Deeply Infiltrating endometriosis: Implications, diagnosis, and management. Obstet Gynecol Surv. 2001;56(3):168-77. Doi: https://doi. org/10.1097/00006254-200103000-00024

26. Donnez J, Nisolle M, Gillerot S, Smets M, Bassil S, Casanas-Roux F. Recto vaginal septum adenomyotic nodules: A series of 500 cases. BJOG An Int J Obstet Gynaecol. 1997;104(9):1014-8. Doi: https://doi. org/10.1111/j.1471-0528.1997.tb12059.x

27. Chapron C, Dubuisson JB. Laparoscopic treatment of deep endometriosis located on the uterosacral ligaments. Hum Reprod. 1996;11(4):868-73. Doi: https://doi. org/10.1093/oxfordjournals.humrep.a019268 


\section{CONTRIBUCIÓN DE LOS AUTORES}

Claudia Patricia Zambrano-Moncayo: recolección de datos, valoración de pacientes, análisis de datos, redacción del manuscrito.

Ana Lucía Herrera-Betancourt: recolección de datos.

Lina María Zuluaga-García: recolección de datos. Danny Leandro Piedrahíta-Gutiérrez: recolección de datos.
Juan Diego Villegas-Echeverri: valoración de pacientes, realización de la cirugía, análisis de datos, redacción del manuscrito.

José Duván López-Jaramillo: valoración de pacientes, realización de la cirugía.

Jorge Darío López-Isanoa: valoración de pacientes, realización de la cirugía.

Claudia Bastidas-Guarín: análisis de datos, redacción del manuscrito.

Juan José Montoya-Martínez: redacción del manuscrito. 